\title{
Dynamic bayesian updating approach for predicting bridge condition based on Indonesia- bridge management system (I-BMS)
}

\author{
Jojok Widodo Soetjipto ${ }^{1,2, *}$, Tri Joko Wahyu Adi $^{1}$, and Nadjadji Anwar ${ }^{1}$ \\ ${ }^{1}$ Department of Civil Engineering, Institut Teknologi Sepuluh Nopember, Surabaya, Indonesia \\ ${ }^{2}$ Department of Civil Engineering, The University of Jember, Jember, Indonesia
}

\begin{abstract}
Bridges are one of the most important infrastructures which support the transportation system. It requires continuous monitoring to keep its condition and functionality. Bridge monitoring is used to support the maintenance strategy in order to prevent deterioration and sudden failure. This paper aims to propose a probabilistic prediction model of bridge conditions based on the Dynamic Bayesian Updating Approach. Around 3.166 data of bridges in Indonesia were collected from the Directorate of Bridges of the Ministry of Public Works and Housing for calculating the conditional probability table (CPT) of the model. A medium-span concrete bridge was used as a case study to validate the proposed model. The results show that the proposed model can predict the condition of the bridge accurately. It also can be used as an early warning system in order to prevent disasters due to technology failure.
\end{abstract}

\section{Introduction}

Indonesia, as a developing country, provides infrastructures such as roads and bridges for equitable development across its territory. Indonesia has built more than 89,000 bridges, which connect areas separated by rivers, valleys, and seas to meet the economic needs of each region.

For this purpose, the government prepares a budget for infrastructure development and maintenance. However, maintenance budgets fall into the low category due to limited budget availability. As a result, infrastructure maintenance has not been properly implemented.

Especially for bridges, the government issued the Indonesia-Bridge Management System (I-BMS) to monitor the condition of bridges and to make bridges a maintenance priority. Research on the application of the BMS has been widely used, in which the BMS can be used as tool to predict the condition of bridges' reinforced concrete girders [1], to evaluate the performance and remaining life of bridges [2], [3], and to predict the residual life of bridges and its damages [4]. In 2016, Widodo, Adi and Anwar also proposed a bridge failure prediction model based on System Dynamic [5]. However, those models have not been able to solve the bridge condition prediction problem which accommodates the

\footnotetext{
* Corresponding author: jojok.teknik@unej.ac.id
} 
relationship among bridge components. Therefore, this research will develop a temporal prediction method for bridge conditions by considering the effect of bridge components' conditions based on the Dynamic Bayesian Updating Approach. The proposed model is expected to eliminate the weakness of the previous methods.

\section{BMS data mining}

BMS data mining is a method to find the pattern of a BMS dataset which were observed from a very large number of bridges within a certain time. BMS data mining is designed to scan databases aimed to find useful new information that has not been previously known. The BMS data mining scheme can be seen in Figure 1.

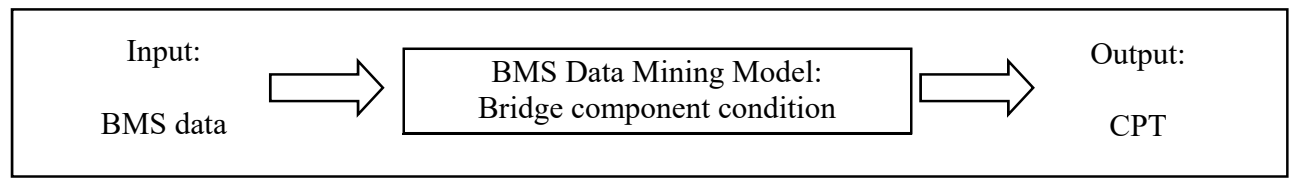

Fig. 1. BMS data mining model.

This research used BMS data, 3.166 bridges across Indonesia, compiled by Directorate of Bridges, Directorate General of Highways, Ministry of Public Works and Housing. The selected bridges are reinforced-concrete girder bridge with spans of 10 to 25 meters. The BMS data consist of bridge component conditions which were observed from the year 2013 to 2015 . For the purpose of modelling and testing, these data were divided into 2 parts, i.e. 2.533 data $(80 \%)$ are used for the model, while the rest is used for model testing/validation.

The condition of each bridge component is obtained from the BMS value (0-5) which has been changed into probability rating and condition categories ("Good", "Moderate" and "Fail"). This method refers to the results of previous research (Markov-System DynamicMSD method) for prediction of conditions and relationships among bridge components [1].

The CPT (Conditional Probability Table) is obtained from the probability of each bridge component condition and joint probability between the bridge and its components. For the Dynamic Bayesian model, joint probability is made for each component in the period of time $t_{n-1}$ to $t_{n}$ (according to the time slice required).

\section{Bayesian}

The Bayesian model can be implemented to predict the probability of an event based on previous events. Many studies on Bayesian applications have been conducted including seismic hazard studies [6], prediction of soil corrosivity [7], forensic assessment of bridge collapse [8] and others. The Bayesian model uses Bayes's theory as follows [9]:

$$
\begin{aligned}
& p(\theta \mid y)=\frac{p(\theta, y)}{p(y)}=\frac{p(\theta) p(y \mid \theta)}{p(y)} \\
& p(y)=\int p(\theta) p(y \mid \theta) d \theta
\end{aligned}
$$

where $p(\theta)$ and $p(y)$ are prior and posterior probability; $P(y \mid \theta)$ is the likelihood function or probability y given $\theta$.

\subsection{Direct acyclic graph (DAG)}

The Bayesian model is a graphical model that makes a probabilistic relationship among variables. The graphic is arranged in a DAG to connect a node (stochastic variable) with 
others directly. To construct a DAG, please refer to the result of the MSD study [1]. To facilitate the making of a DAG structure, this study uses GeNIe 2.1 software (see Figure 2).
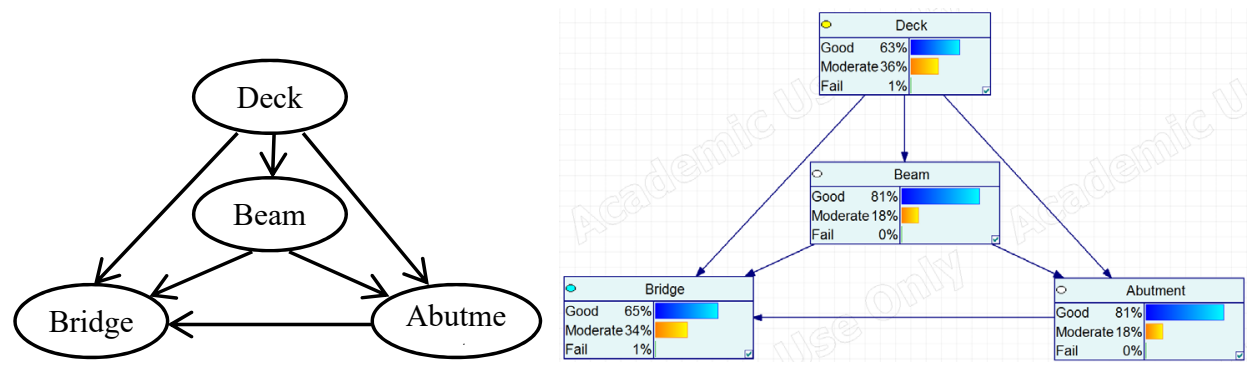

Fig. 2. BMS Bayesian Network (BNs) proposed.

\subsection{Conditional probability table (CPT)}

After the DAG is completed, the Bayesian Network (BNs) requires a CPT to determine the probability of an event. The CPT is calculated based on joint probability among nodes according to equations (1) and (2). To arrange the CPT, several stages need to be conducted:

Step1: Verifying the I-BMS data especially Reinforced-Concrete Bridge with spans of 10 to 25 metres

Step 2: Giving random numbers on each bridge data and then sorting its data to divide into 2 groups, i.e. $80 \%$ data for the model and $20 \%$ for data testing.

Step 3: CPT is calculated based on the 80\% data model using the formulas (1) and (2).

Based on the Indonesian BMS, the condition of bridges can be measured using Visual Inspection which is represented by a Score from 0 to 5. A Score of 0-1 means "Good condition", a Score of 2-3 means "Moderate condition", and a Score of 4-5 means "Fail condition". A recapitulation of CPT for Deck, Beam, Abutment, and Bridge are listed in Tables 1 to 4 .

Table 1. CPT deck.

\begin{tabular}{|c|c|}
\hline Deck & Probability \\
\hline G & 0.632 \\
\hline M & 0.357 \\
\hline F & 0.011 \\
\hline
\end{tabular}

Table 2. CPT Beam.

\begin{tabular}{|c|c|c|c|c|}
\hline & Deck & G & M & F \\
\hline \multirow{3}{*}{ Beam } & G & 0.873 & 0.707 & 0.753 \\
\cline { 2 - 5 } & M & 0.123 & 0.289 & 0.141 \\
\cline { 2 - 5 } & F & 0.003 & 0.004 & 0.106 \\
\hline
\end{tabular}

Table 3. CPT abutment.

\begin{tabular}{|c|c|c|c|c|c|c|c|c|c|c|}
\hline \multicolumn{2}{|c|}{ Deck } & \multicolumn{3}{c|}{ G } & \multicolumn{3}{c|}{ M } & \multicolumn{3}{c|}{ F } \\
\hline \multicolumn{2}{|c|}{ Beam } & G & M & F & G & M & F & G & M & F \\
\hline \multirow{3}{*}{ Abutment } & G & 0.857 & 0.720 & 1.000 & 0.825 & 0.626 & 0.417 & 0.766 & 0.833 & 0.556 \\
\cline { 2 - 10 } & M & 0.142 & 0.279 & 0.000 & 0.172 & 0.369 & 0.250 & 0.203 & 0.083 & 0.111 \\
\cline { 2 - 10 } & F & 0.001 & 0.002 & 0.000 & 0.003 & 0.005 & 0.333 & 0.031 & 0.083 & 0.333 \\
\hline
\end{tabular}


Table 4. CPT bridge.

\begin{tabular}{|c|c|c|c|c|c|c|c|c|c|c|}
\hline \multirow{3}{*}{\multicolumn{2}{|c|}{\begin{tabular}{|c|} 
Deck \\
Beam \\
Abutment \\
\end{tabular}}} & \multicolumn{9}{|c|}{ G } \\
\hline & & \multicolumn{3}{|c|}{ G } & \multicolumn{3}{|c|}{ M } & \multicolumn{3}{|c|}{$\mathbf{F}$} \\
\hline & & G & M & $\mathbf{F}$ & G & M & $\mathbf{F}$ & G & M & $\mathbf{F}$ \\
\hline \multirow{3}{*}{ Bridge } & $\mathrm{G}$ & 0.962 & \begin{tabular}{|l|}
0.000 \\
\end{tabular} & 0.000 & 0.000 & 0.000 & 0.000 & 0.000 & 0.000 & 0.000 \\
\hline & $\bar{M}$ & 0.037 & 1.000 & 0.000 & 1.000 & 1.000 & 0.000 & 0.000 & 0.000 & 0.000 \\
\hline & $\mathrm{F}$ & 0.001 & 0.000 & 1.000 & 0.000 & 0.000 & 1.000 & 1.000 & 1.000 & 1.000 \\
\hline \multicolumn{2}{|c|}{ Deck } & \multicolumn{9}{|c|}{$\mathbf{M}$} \\
\hline \multicolumn{2}{|c|}{ Beam } & \multicolumn{3}{|c|}{ G } & \multicolumn{3}{|c|}{ M } & \multicolumn{3}{|c|}{$\mathbf{F}$} \\
\hline \multicolumn{2}{|c|}{ Abutment } & G & M & $\mathbf{F}$ & G & $\mathbf{M}$ & $\mathbf{F}$ & G & M & $\mathbf{F}$ \\
\hline \multirow{3}{*}{ Bridge } & $\mathrm{G}$ & 0.956 & 0.000 & 0.000 & 0.000 & 0.000 & 0.000 & 0.000 & 0.000 & 0.000 \\
\hline & $\mathrm{M}$ & 0.043 & 1.000 & 0.000 & 1.000 & 1.000 & 0.000 & 0.000 & 0.000 & 0.000 \\
\hline & $\mathrm{F}$ & 0.001 & 0.000 & 1.000 & 0.000 & 0.000 & 1.000 & 1.000 & 1.000 & 1.000 \\
\hline \multicolumn{2}{|c|}{ Deck } & \multicolumn{9}{|c|}{$\mathbf{F}$} \\
\hline \multicolumn{2}{|c|}{ Beam } & \multicolumn{3}{|c|}{ G } & \multicolumn{3}{|c|}{ M } & \multicolumn{3}{|c|}{$\mathbf{F}$} \\
\hline \multicolumn{2}{|c|}{ Abutment } & $\mathbf{G}$ & M & $\mathbf{F}$ & $\mathbf{G}$ & $\mathbf{M}$ & $\mathbf{F}$ & G & M & $\mathbf{F}$ \\
\hline \multirow{3}{*}{ Bridge } & $\mathrm{G}$ & 0.000 & 0.000 & 0.000 & 0.000 & 0.000 & 0.000 & 0.000 & 0.000 & 0.000 \\
\hline & M & 0.959 & 1.000 & 0.000 & 1.000 & 1.000 & 0.000 & 0.000 & 0.000 & 0.000 \\
\hline & $\mathrm{F}$ & 0.041 & 0.000 & 1.000 & 0.000 & 0.000 & 1.000 & 1.000 & 1.000 & 1.000 \\
\hline
\end{tabular}

\subsection{Dynamic bayesian network (DBN)}

The DBN consists of several parts of the BNs, each of it representing a system in a slice of time $(0,1, \ldots, n)$. DBN can show the evolution of the model probability from the time (n-1) to $\mathrm{n}$ and so on (see Figure 3).

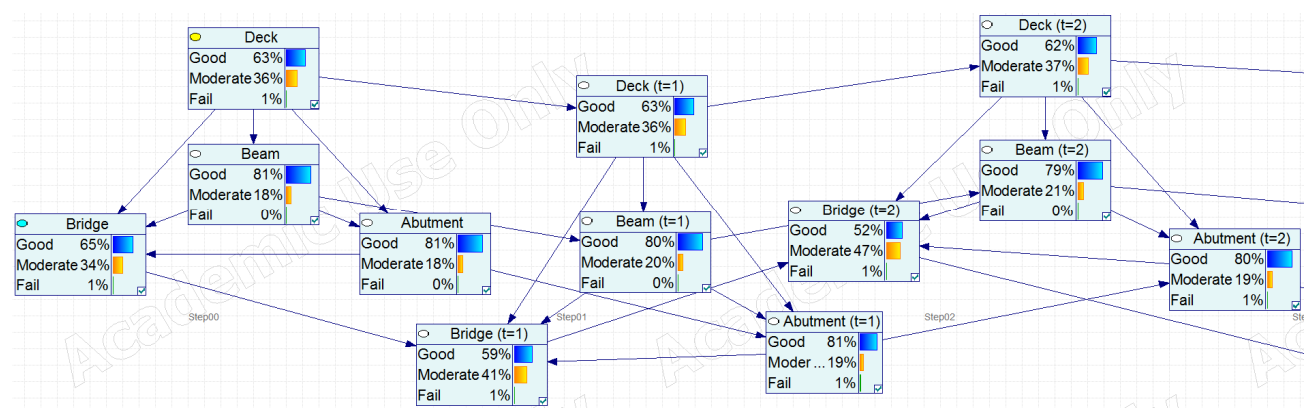

Fig. 3. Representation of the DBN prediction model.

\section{Results and discussions}

This study uses a DBN model with a temporal plate (10 slices in time). Furthermore, the data is simulated using GeNIe 2.1 software to determine the bridge condition behavior (see Figure 4). In this section, the discussion will be divided into: (i) simulation; (ii) validation; and (iii) scenarios. 


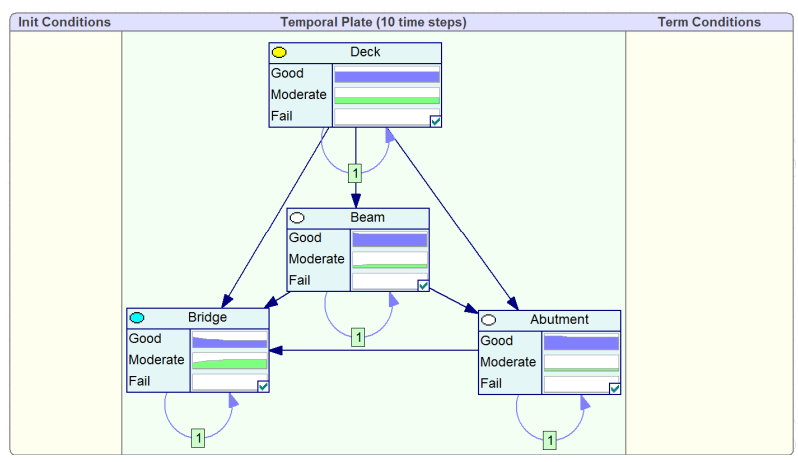

Fig. 4. The DBN prediction models.

\subsection{Simulation model}

To perform the simulation, the DBN model's data is given based on the CPT value obtained from the I-BMS data. The result of the simulation can be seen in Figure 5 which is interpreted as follows: (i) Probability of Bridge is strongly influenced by the probability of Beam and Abutment. This is shown by the decreasing probability of "Good" on the Bridge due to the decreasing probability of "Good" on both Beam and Abutment; (ii) Probability of Deck has a very small effect on the probability of Bridge. This can be seen from the probability of "Good" on the Deck being relatively fixed, but which did not influence the probability of "Good" of the Bridge.

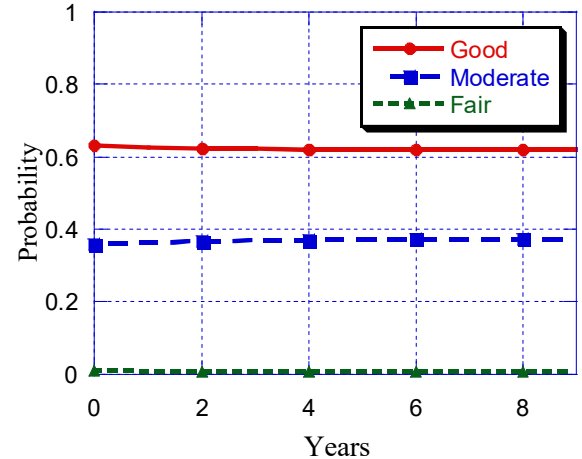

(a) DBN Deck

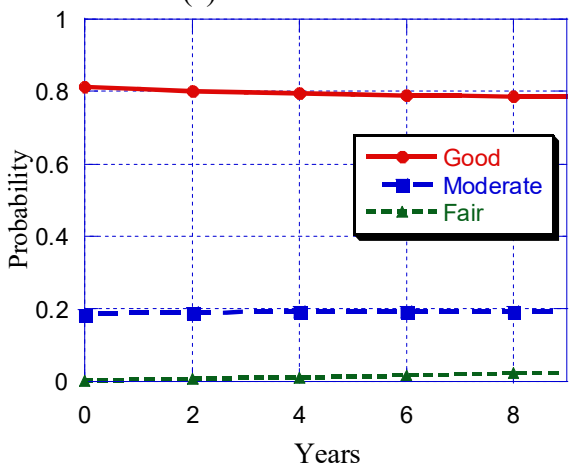

(c) DBN Abutment

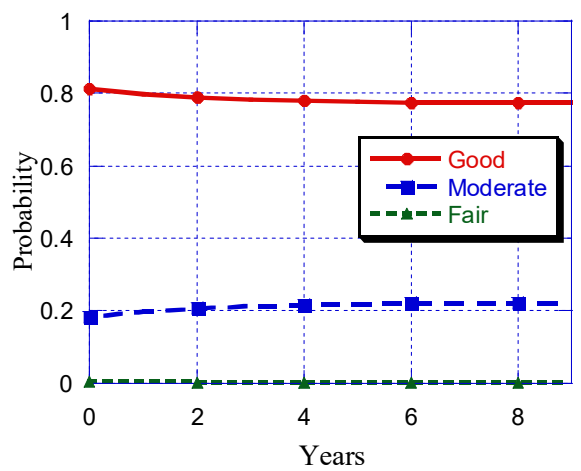

(b) DBN Beam

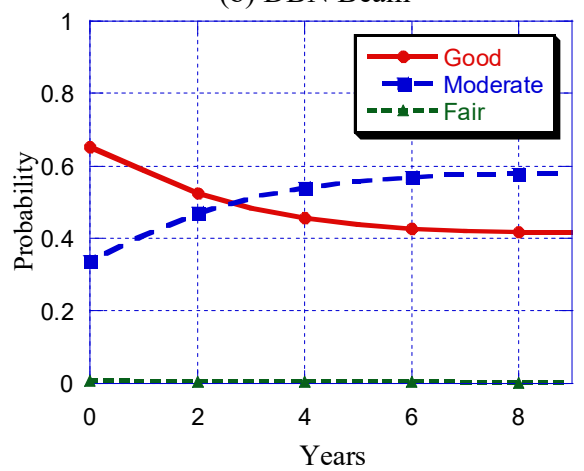

(d) DBN Bridge

Fig. 5. The output of DBN model for the bridge and its components. 


\subsection{Validation model}

To validate the model and calculate the model accuracy, a "match/ no match" approach is used [10]. The model will compare the result of the proposed model with benchmark data (condition of the bridge component base of I-BMS), in terms of their respective classification ("match" or "no match").

20 bridges in the data testing will be applied to the DBN model, then to be compared with the BMS observation data (benchmark). The result of the model validation is shown in Table 5. The result shows that the DBN model can be applied to determine current (year 0) bridge conditions with $100 \%$ accuracy. However, the level of accuracy decreases on the second year and third year up to $80 \%$.

Table 5. Validation model.

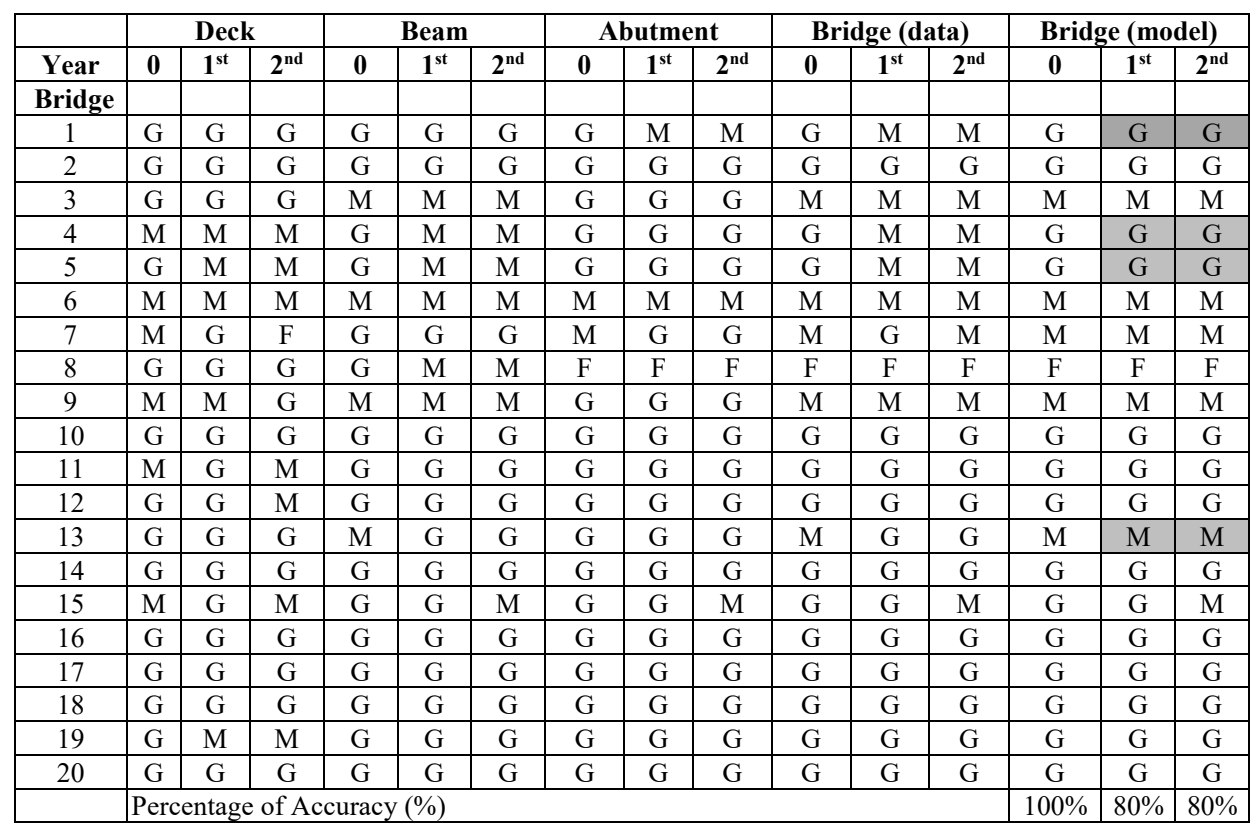

\subsection{Scenario model}

To study the effect of behavior changes of bridge component conditions, the DBN model will be set in 3 scenarios (see Table 6).

Table 6. Scenario model.

\begin{tabular}{|c|c|c|c|}
\hline Scenario & Deck & Beam & Abutment \\
\hline \multirow{3}{*}{1} & $\mathrm{G}$ & $\mathrm{G}$ & $\mathrm{G}$ \\
\cline { 2 - 4 } & $\mathrm{M}$ & $\mathrm{G}$ & $\mathrm{G}$ \\
\cline { 2 - 4 } & $\mathrm{F}$ & $\mathrm{G}$ & $\mathrm{G}$ \\
\hline \multirow{3}{*}{2} & $\mathrm{G}$ & $\mathrm{G}$ & $\mathrm{G}$ \\
\cline { 2 - 4 } & $\mathrm{G}$ & $\mathrm{M}$ & $\mathrm{G}$ \\
\cline { 2 - 4 } & $\mathrm{G}$ & $\mathrm{F}$ & $\mathrm{G}$ \\
\hline \multirow{3}{*}{3} & $\mathrm{G}$ & $\mathrm{G}$ & $\mathrm{G}$ \\
\cline { 2 - 4 } & $\mathrm{G}$ & $\mathrm{G}$ & $\mathrm{M}$ \\
\cline { 2 - 4 } & $\mathrm{G}$ & $\mathrm{G}$ & $\mathrm{F}$ \\
\hline
\end{tabular}


The first scenario: The Deck condition is changed (from "Good" to "Fail") while the Beam and the Abutment have a fixed condition ("Good"). The results of scenario 1 can be seen in Figure 6. The result shows that the Bridge condition is "Good" even though the condition of the Deck is "Moderate". Nevertheless, the condition of the Bridge changes to "Moderate" due to the Deck condition changing to "Fail". It can be inferred that the influence of the Deck condition on the Bridge condition is relatively small. The probability of the Bridge condition is greatly influenced by the temporal CPT. So that, it appears on decreasing or increasing on the Bridge condition every year.
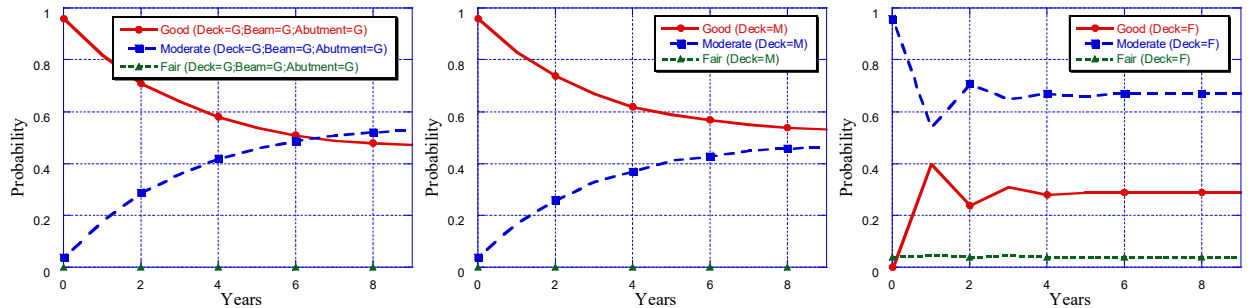

Fig. 6. Bridge condition on scenario 1 .

The second scenario: The Beam condition was changed (from "Good" to "Fail") while both the Deck and Abutment were set up in "Good" condition only. The results of scenario 2 can be shown in Figure 7. The Bridge condition is strongly influenced by the Beam condition even though the deck and abutment conditions are "Good". This indicates that the effect of the Beam condition on the Bridge condition is very dominant.
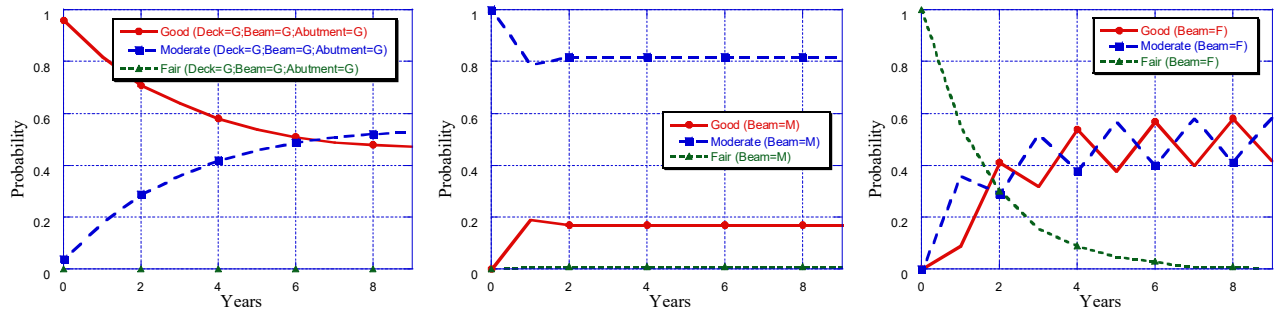

Fig. 7. Bridge condition on scenario 2.

The third scenario: The model is set up to change the Abutment condition (from "Good" to Fail") while the Deck and Beam have a fixed condition (i.e. "Good"). Bridge condition strongly influenced by Abutment condition even though the Deck and Beam condition is "Good" (see Figure 8). It can be concluded that the effect of the Abutment condition is very dominant in the Bridge condition. In this case, the Abutment condition changed to "Fail", and has an anomaly condition at the slice in time of the second year and later. This anomaly condition is estimated due to limited data changes in the Fail's Bridge condition.
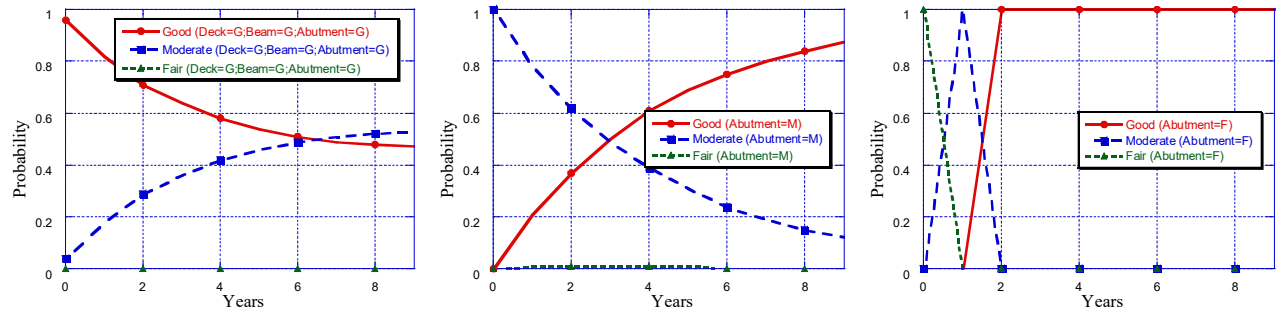

Fig. 8. Bridge condition on scenario 3. 
From the perspective of bridge safety, to prevent the sudden collapse of the bridge, more serious attention should be undertaken when handling Beams and Abutments rather than the Deck, because the Beam and Abutment have a significant influence on the Bridge condition. However, from the bridge's user convenience perspective, a good deck condition is indispensable for driving comfort. For the maintenance strategy, it is very important to prioritize the repair of bridge components (Beam or Abutment) due to the limited budget of the government.

\section{Conclusion}

The Dynamic Bayesian Updating Approach can be used to assess the Bridge condition accurately. The simulation shows that each bridge component contributes to determining the Bridge condition. The model scenarios also provide clarity that the effect on the Bridge condition is provided by, from largest to smallest, the Abutment, Beam and Deck.

The Dynamic Bayesian Updating Approach can also be used as a guide for the maintenance and operation strategy of the bridge. To prevent the sudden collapse of the bridge, the government should pay very serious attention to the damage protection of abutments and beams. Finally, the proposed model can also be used as an early warning system to prevent bridge failure, even though the model accuracy still needs to be improved.

The author would like to thank the Directorate of Bridges, Directorate General of Bina Marga, the Ministry of Public Works and Housing for providing data, manuals, and other needed information for this study. The author also extends deep gratitude and appreciation to Ministry of Research, Technology and Higher Education for funding this research based on the research cooperation 2018.

\section{References}

1. J. W. Soetjipto, T. J. W. Adi, and N. Anwar, Matec Web Conf., 138, 5001 (2017)

2. H. Emoto, J. Takahashi, R. Widyawati, and A. Miyamoto, Procedia Eng., 95, 65 (2014)

3. S. Hasan, S. Setunge, D. W. Law, and Y. Koay, Int. J. Eng. Technol., 7, 1 (2015)

4. G. P. Bu, J. H. Lee, H. Guan, Y. C. Loo, and M. Blumenstein, J. Perform. Constr. Facil., 29, 3 (2015)

5. J. W. Soetjipto, T. J. W. Adi, and N. Anwar, Int. J. Eng. Technol. Innov., 6, 4 (2016).

6. J. P. Wang, Applications of Statistics and Probability in Civil Engineering, 12, MS12 (2015)

7. G. Demissie and S. Tesfamariam, Applications of Statistics and Probability in Civil Engineering, 12, MS1 (2015)

8. J. Marková, M. Sy, and M. Holicky, Eng. Fail. Anal. J., 30, 1-9 (2013)

9. A. Gelman, J. B. Carlin, H. S. Stern, D. B. Dunson, A. Vehtari, and D. B. Rubin, Bayesian Data Analysis, Boca Raton: CRC Press Taylor \& Francis Group (2014)

10. S. Leu and W. Joko, Tri Adi, Eng. Appl. Artif. Intell., 24, 4, 658-665 (2011) 